\title{
Sensory Evaluation of Vegetable-Infused Fruit-Flavored Applesauce and the Comparison between Adults and Children
}

\author{
Peter Bordi ${ }^{*}$, Kiwon Lee, Martha Conklin \\ School of Hospitality Management, Pennsylvania State University, University Park, USA. \\ Email: *PLBJr@psu.edu
}

Received December $17^{\text {th }}, 2012$; revised March 24 $4^{\text {th }}, 2013$; accepted April $4^{\text {th }}, 2013$

Copyright (C) 2013 Peter Bordi et al. This is an open access article distributed under the Creative Commons Attribution License, which permits unrestricted use, distribution, and reproduction in any medium, provided the original work is properly cited.

\begin{abstract}
Low consumption of fruits and vegetables among children is considered to be a leading contributor to childhood obesity, making it prudent to develop healthier foods that are accessible, affordable and satisfying to children. Our objective was to investigate the sensory characteristics and preferred flavors of vegetable-infused applesauce among adults (male and female) and $7^{\text {th }}$ grade middle school students; and to compare the flavor preferences of adults and children in order to gather information that helps determine target demographics for each applesauce flavor. Sensory testing for eight attributes (overall liking, overall appearance, appearance of texture, appearance of color, taste, mouth feel, amount of fruit flavor, amount of sweetness) were rated by 120 adults and 106 middle school students. Adults and kids demonstrated significantly different preferences for the tropical and mixed berry applesauce flavors but no difference with the strawberry banana-flavored applesauce. Female adults demonstrated clear preference for the strawberry banana flavor; children demonstrated a higher preference for the mixed berry flavor, followed by the tropical flavor. Overall, both adults and children rated all of the applesauce flavors favorably, suggesting that developing and bringing such products to market could be an effective method for helping adults and children consume more fruits and vegetables.
\end{abstract}

Keywords: Vegetable-Infused Applesauce; Stealth Health Foods; Childhood Obesity; Fruit and Vegetable Consumption

\section{Introduction}

Obesity among children has reached epidemic proportions [1]. Using data collected from the 2007-2008 National Health and Nutrition Examination Survey, Ogden and Caroll [2] found that the rate of obesity among preschool children (ages 2 - 5) increased from 5.0\% to $10.4 \%$ between $1976-1980$ and $2007-2008$; the rate among children ages 6 - 11 increased from $6.5 \%$ to $19.6 \%$ during the same period; and the rate among adolescents ages 12 - 19 increased from 5.0\% to $18.1 \%$.

Not surprisingly, children who are overweight are more susceptible to various physical ailments. For example, two-thirds of overweight young people demonstrate at least one additional risk factor for heart disease, such as high cholesterol or high blood pressure [3]. Children who are overweight also are at greater risk for type 2 diabetes, bone and joint problems, sleep apnea and social

${ }^{*}$ Corresponding author. discrimination [4,5]. Compounding the problem is the fact that overweight in childhood often persists into adulthood; as a result, overweight children are at greater risk of becoming overweight or obese adults, suffering from health problems such as type 2 diabetes, several types of cancer or heart disease in their adult years [6].

Low consumption of fruits and vegetables among children is considered to be a leading contributing factor to childhood obesity. The US Department of Agriculture [7] recommends that girls aged 9 - 18 years consume 1.5 cups of fruits or 2 - 2.5 cups of vegetables daily, and boys aged 9 - 18 years consume 1.5 - 2 cups of fruits or 2.5 - 3 cups of vegetables daily; however, children's daily fruit and vegetable intake does not meet the USDA's recommendations [8]. The State Indicator Report on Fruits and Vegetables [9] showed that only 5\% $11 \%$ of US youth in grades $9-12$ (14 - 18 years old on average) consume two (1 cup) or more servings of fruit and three (1.5 cups) or more servings of vegetables per 
day, with an assumption that a serving equals $1 / 2$ cup. The percentage of children in grades $9-12$ that consumed fruit two or more times per day ranged from $21.3 \%$ to $34.5 \%$, while the percentage of the same-aged children that consumed vegetables three or more times per day ranged from $8.8 \%$ to $15.8 \%$. Lorson and her colleagues [10] also found that children and adolescents consumed low amounts of fruits and vegetables compared to the USDA recommendations, especially boys and older children.

Dennison, Rockwell and Baker [11] also noted the lower intake of vegetables compared to fruits among preschool-aged children. The results showed that preschool-aged children consumed about $80 \%$ of the recommended fruit servings per day ( 1 to 1.5 cups per day (USDA, 2011a)), but consumed only $25 \%$ of the recommended vegetable servings per day ( 1 to 1.5 cups per day [7]). In another study [12], only 30\% of children aged 2 19 years met the consumption recommendations for fruits, while only $36 \%$ of children aged 2 - 19 years met the consumption recommendations for vegetables.

Due to the benefits of a high fruit and vegetable intake, including a promotion of health and a prevention of chronic disease [13,14], several school- or family-based intervention programs have been introduced in an effort to increase fruit and vegetable consumption among children. For example, a recent nationwide campaign named "Let's Move" [15] offers grants to schools to encourage them to provide salad bars with more fruits and vegetables in their cafeterias. However, very few studies have been conducted on commercial food products with fruits and vegetables as ingredients in order to gauge their effectiveness at increasing fruit and vegetable consumption among children.

Apples, on the other hand, are a widely consumed, rich source of phytochemicals; epidemiological studies have linked the consumption of apples with reduced risk of some cancers, cardiovascular disease, asthma, and diabetes. In the laboratory, apples have been found to have very strong antioxidant activity, inhibit cancer cell proliferation, decrease lipid oxidation, and lower cholesterol [16]. Because of this, commercial food products which include apples as an ingredient-applesauce, for example, are often considered to be a healthy and convenient snack, especially for children. Additionally, applesauce is also one of the foods included in the BRAT (bananas, rice, applesauce and toast) or CRAM (cereal, rice, applesauce and milk) diets historically recommended for those suffering from diarrhea, dyspepsia, gastroenteritis, and/or other gastrointestinal distress. As several reports $[17,18]$ that calculated nutrition quality of applesauce showed, applesauce delivers fiber, vitamin $\mathrm{C}$ and energy-providing B vitamins.
Interventions that increase vegetable intake among children need to be considered more seriously [11]. One possible method is by including vegetables as ingredients in "stealth health foods", or foods that are more commonly accepted and consumed by consumers that include other foods/ingredients that aren't as acceptable to consumers despite their nutritional value [19,20]. Based on the premise that children eat applesauce with little resistance, it would be meaningful to evaluate the sensory characteristics of vegetable-infused applesauce as a stealth food. Having children eat vegetables unconsciously while consuming applesauce might help them develop a sense of familiarity with the taste, and subsequently increase their acceptance of vegetables.

It's apparent that adults (e.g., parents) select foods including snacks for their children and that adults and children have different preferences for food items. So, it would be meaningful to investigate different preferences of adults and children, respectively, in order to figure out the acceptability and the marketability.

The purpose of this study, therefore, was to investigate the sensory characteristics and preferred flavors of vegetable-infused applesauce among adults (male and female) and children; and to compare the flavor preferences of adults and children in order to gather information that helps determine target demographics for each applesauce flavor.

\section{Materials and Methods}

\subsection{Materials}

The Center for Food Innovation (CFI), located on the campus of a large northeastern university, provided the facilities for this study. All of the applesauce used for the study was produced by Knouse Foods; flavors tested included tropical, mixed berry and strawberry. Each full serving of the tested applesauce contained one full serving of fruits and one full serving of vegetables. In addition to apples and apple concentrate (common ingredients), tropical flavor contains peaches and natural peach flavor to generate the tropical flavor, and carrot and cucumber concentrates for the vegetable servings. Mixed berry flavor contains strawberry, raspberry, and blueberry purees to generate mixed berry flavor, and purple carrot and cucumber concentrates for the vegetable servings. Strawberry flavor contains only strawberry puree, and carrot and cucumber concentrates. The ingredients and nutritional information for each applesauce flavor are described in Figure 1.

Compared to the "regular" applesauce brands already available in grocery stores, the vegetable-infused Knouse applesauces tested contained fewer calories, less sugar, more dietary fiber, and more vitamins (vitamins A and C) 
Tropical

Nutrition Facts

Serving Size (100g)

Servings Per Container

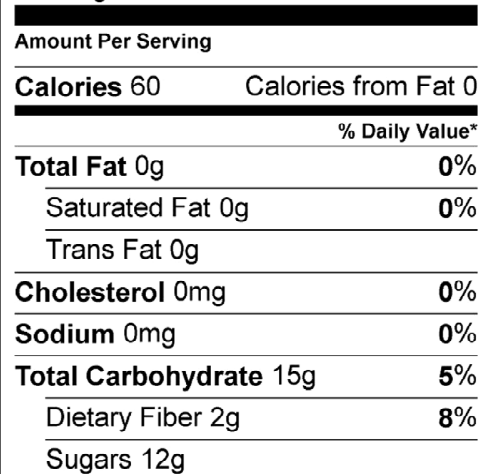

\section{Protein $\mathrm{Og}$}

Vitamin A 15\% $\quad$ V Vitamin C 110\%

Calcium $0 \% \quad$ - Iron $0 \%$

${ }^{*}$ Percent Daily Values are based on a 2,000 calorie diet. Your daily values may be higher or lower

depending on your calorie needs:

\begin{tabular}{llll}
\multicolumn{4}{c}{ depending on your calorie needs: } \\
& Calories: & 2,000 & 2,500 \\
\hline Total Fat & Less than & $65 \mathrm{~g}$ & $80 \mathrm{~g}$ \\
Saturated Fat & Less than & $20 \mathrm{~g}$ & $25 \mathrm{~g}$ \\
Cholesterol & Less than & $300 \mathrm{mg}$ & $300 \mathrm{mg}$ \\
Sodium & Less than & $2,400 \mathrm{mg}$ & $2,400 \mathrm{mg}$ \\
Total Carbohydrate & $300 \mathrm{~g}$ & $375 \mathrm{~g}$ \\
Dietary Fiber & $25 \mathrm{~g}$ & $30 \mathrm{~g}$ \\
\hline Calories per gram: & & \\
\multicolumn{2}{l}{ Fat 9 } & Carbohydrate 4 & - Protein 4 \\
\hline
\end{tabular}

INGREDIENTS: Apples, Peaches, water, Cucumber concentrate, apple concentrate, Carrot Concentrate, Natural Peach Flavor, Ascorbic Acid, Citric Acid.
Mixed Berry

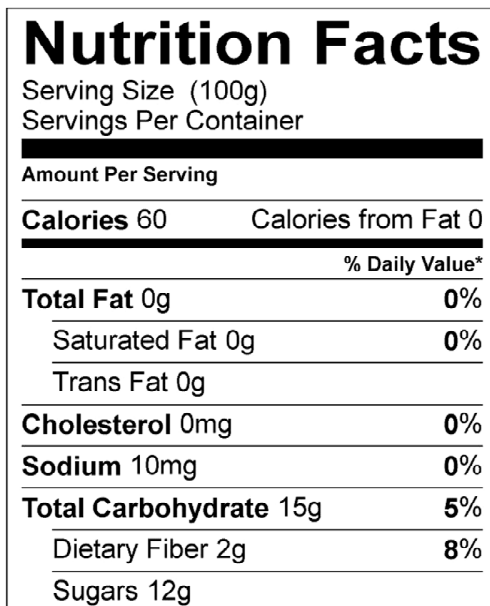

\section{Protein Og}

\begin{tabular}{|c|c|c|c|}
\hline Vitamin A 0\% & \multicolumn{3}{|c|}{ - Vitamin C $120 \%$} \\
\hline Calcium 2\% & \multicolumn{3}{|c|}{ - Iron $2 \%$} \\
\hline \multicolumn{4}{|c|}{$\begin{array}{l}\text { *Percent Daily Values are based on a } 2,000 \text { calorie } \\
\text { diet. Your daily values may be higher or lower } \\
\text { depending on your calorie needs: } \\
\begin{array}{l}\text { Calories: } \quad 2,000 \\
\quad 2,500\end{array}\end{array}$} \\
\hline $\begin{array}{l}\text { Total Fat } \\
\text { Saturated Fat } \\
\text { Cholesterol } \\
\text { Sodium } \\
\text { Total Carbohydra } \\
\text { Dietary Fiber }\end{array}$ & $\begin{array}{l}\text { Less than } \\
\text { Less than } \\
\text { Less than } \\
\text { Less than } \\
\text { te }\end{array}$ & $\begin{array}{l}65 \mathrm{~g} \\
20 \mathrm{~g} \\
300 \mathrm{mg} \\
2,400 \mathrm{mg} \\
300 \mathrm{~g} \\
25 \mathrm{~g} \\
\end{array}$ & $\begin{array}{l}80 \mathrm{~g} \\
25 \mathrm{~g} \\
300 \mathrm{mg} \\
2,400 \mathrm{mg} \\
375 \mathrm{~g} \\
30 \mathrm{~g} \\
\end{array}$ \\
\hline $\begin{array}{c}\text { lories per gra } \\
\text { Fat } 9\end{array}$ & ohy & $\mathrm{P}$ & \\
\hline
\end{tabular}

INGREDIENTS: Apples, water, Purple carrot juice concentrate, apple concentrate,

strawberry puree, raspberry puree, Blueberry Puree, Cucumber concentrate, Natural flavor, Ascorbic Acid, Citric Acid.
Strawberry

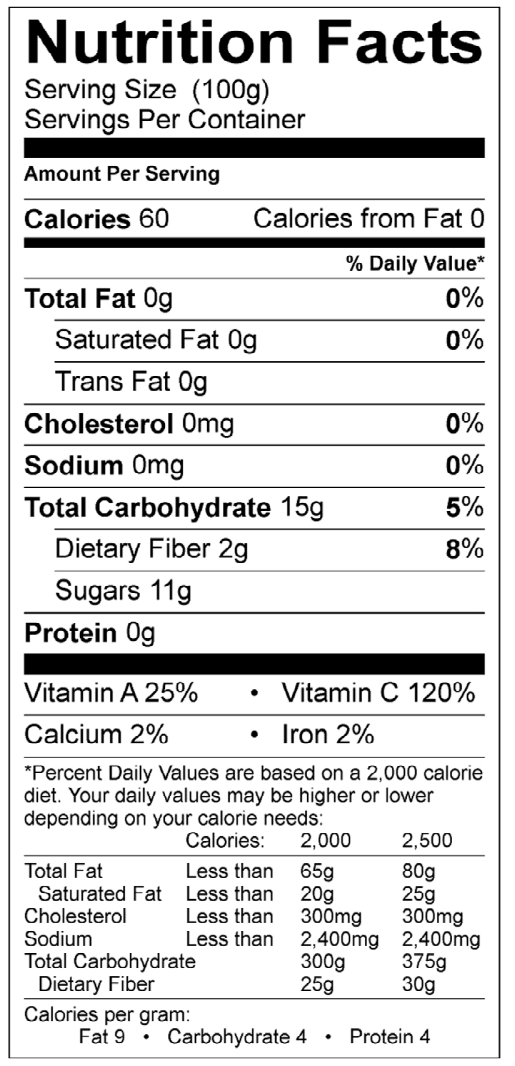

INGREDIENTS: Apples, strawberry puree, water, Cucumber concentrate, apple concentrate, Carrot Concentrate, natural flavor, natural color, Ascorbic Acid, Citric Acid.

Figure 1. Applesauce ingredients and nutritional information.

and minerals (calcium and iron). One brand of "regular" applesauce available in grocery stores that had apples, high fructose corn syrup, corn syrup, water, and ascorbic acid as ingredients contains 70.8 calories, $1.8 \mathrm{~g}$ dietary fiber, 14.2 g sugars, and no vitamins or minerals per 100 g. Another mixed berry applesauce with apples, high fructose corn syrup, corn syrup, strawberry puree, red raspberry puree, blueberry puree, natural flavors, water, ascorbic acid, and erythorbic acid contains the same amount of calories, dietary fiber, and sugars as the "regular" brand as well as $100 \%$ of the daily recommended value of vitamin C per $100 \mathrm{~g}$.

Compared to Knouse's “regular” applesauce, which also already is available in grocery stores, the vegetableinfused Knouse applesauces contain more calories and sugar; Knouse’s "regular” applesauce (which has apples, water, unsweetened apple juice, and ascorbic acid as in- gredients) contains 44.3 calories, $1.8 \mathrm{~g}$ dietary fiber, 7.1 g sugars, and no vitamins or minerals per 100 g (amounts of nutrients were calculated per $100 \mathrm{~g}$ from the original serving size of $113 \mathrm{~g}$ ). This is acceptable because the additional sugars were natural sugars found in fruits and vegetables and were not generated from artificial sources.

\subsection{Procedures}

Sensory testing of the applesauce took place in CFI's sensory laboratory and in a middle school located in the northeastern United States. Panelists included college students, faculty, and staff recruited from the university; and 7th grade students at the middle school in south central Pennsylvania because they are good tasters due to their age. Researchers filled cups with two ounces of applesauce in each flavor of applesauce, with each cup 
being numbered by flavor with a three-digit sample number. Panelists received a sample of each of the three flavors and were asked to evaluate them on overall liking, overall appearance, appearance of texture, appearance of color, taste of applesauce, mouth feel of applesauce, amount of fruit flavor, and amount of sweetness. All attributes were tested on 7-point hedonic scales.

Adult panelists recorded sensory characteristics at one of ten computer stations located in CFI's sensory laboratory. Middle school panelists recorded sensory characteristics at the middle school using paper ballots, and the data were then inputted by hand at one of the computer stations in CFI's sensory laboratory. Researchers visited each classroom and explained what the terms means so they were rating from a unified perspective. Results were analyzed using CompuSense ${ }^{\circledR}$ Five software (Guelph, Ontario, Canada). ANOVA was used to compare mean scores of each flavored applesauce by gender of adults (male versus female), by age (adults versus children) and within each group to examine flavor preference.

\section{Results and Discussion}

A total of 226 individuals completed the sensory test120 adults (26 males and 94 females) ranging in age from 18 to 45 years, and 106 middle school students ranging in age from 11 to 14 years. Participants were asked how frequently they typically consume applesauce. As noted in Table 1, an overwhelming majority (77.5\%) said they consumed applesauce either once a week or once a month.

Evaluations of eight attributes were compared by group for each flavor (Tables 2-4). Table 2 summarizes the attribute comparison of the tropical-flavored applesauce. As noted, there were significant differences between the adults and kids in terms of overall liking, overall appearance, color and taste. Kids tended to have more favorable opinions of the tropical-flavored applesauce than adults in general and female adults in particular.

Table 1. Past applesauce consumption.

\begin{tabular}{ccccc}
\hline & \multicolumn{2}{c}{ Adults } & Kids & Total \\
Frequency & $\begin{array}{c}\text { Male } \\
(\mathrm{n}=26)\end{array}$ & $\begin{array}{c}\text { Female } \\
(\mathrm{n}=94)\end{array}$ & & \\
\cline { 2 - 3 }$(\mathrm{n}=106)$ & & \\
\hline Daily & $0(0.0 \%)$ & $5(5.3 \%)$ & $11(10.4 \%)$ & $16(7.1 \%)$ \\
Once a week & $13(50.0 \%)$ & $25(26.6 \%)$ & $27(25.5 \%)$ & $65(28.8 \%)$ \\
Once a month & $13(50.0 \%)$ & $64(68.1 \%)$ & $33(31.1 \%)$ & $110(48.7 \%)$ \\
Less than & $0(0.0 \%)$ & $0(0.0 \%)$ & $26(24.5 \%)$ & $26(11.5 \%)$ \\
once a month & & & & \\
Never & $0(0.0 \%)$ & $0(0.0 \%)$ & $9(8.5 \%)$ & $9(4.0 \%)$ \\
\hline
\end{tabular}

Both kids and adults found the amount of fruit flavor and the amount of sweetness to be just about right (rating around 4 points).

In terms of the Mixed Berry-flavored applesauce (Table 3), kids and adults had significantly different ratings for overall liking, overall appearance, texture, color, taste, and mouth feel; kids tended to provide higher scores for these six attributes, especially when compared to female adults. Interestingly, kids and male adults showed similar- and higher-ratings for the Mixed Berry-flavored applesauce compared to female adults in terms of overall liking, texture, taste, and mouth feel. It is important to note, however, that female adults still had a favorable opinion of the Mixed Berry flavor overall (as noted by the overall liking rating above 4 points). Both adults and kids also indicated that the flavor and sweetness were just about right, rating both either around or above 4 points.

Table 4 provides a comparison of the eight attributes for the strawberry-flavored applesauce. The differences among these attributes were not as significant as they were with the tropical and mixed berry flavors. Both adults and kids seem to rate the strawberry-flavored applesauce highly, giving average scores above 4 points for overall liking, overall appearance, texture, color, taste, and mouth feel; and average scores around 4 points (just about right) for flavor and sweetness.

Researchers then compared the eight attributes by flavor within each group to see which flavor is preferred by each group; results of that comparison are presented in Table 5. Male adults had a significantly higher preference for the strawberry-flavored applesauce over the tropical-flavored applesauce in terms of overall appearance, and a higher preference for mixed berry and strawberry over tropical in terms of color.

Female adults demonstrated much clearer preferences than male adults, rating the overall appearance, texture, color, and taste of the strawberry-flavored applesauce higher than the other two flavors. Even though the differences were not as significant in the overall liking and mouth feel of the strawberry-flavored applesauce, its flavor still had higher scores than the tropical and mixed berry-flavored applesauces.

Kids clearly demonstrated a higher preference for the mixed berry-flavored applesauce, followed by the tropical flavor. Kids had significantly different opinions in terms of overall liking, overall appearance, color, taste, and mouth feel of all three flavors; however, they found the fruit flavor and sweetness amounts of all three flavors to be just about right (around 4 points).

\section{Conclusions}

According to the US Census Bureau, the average life 
and the Comparison between Adults and Children

Table 2. Attribute comparison of tropical-flavored applesauce (by group).

\begin{tabular}{|c|c|c|c|c|c|}
\hline \multirow{2}{*}{ Attributes } & \multicolumn{2}{|c|}{ Adults } & \multirow{2}{*}{ Kids $(\mathrm{n}=106)$} & \multirow{2}{*}{$F$ value } & \multirow{2}{*}{$p$ value } \\
\hline & Male $(n=26)$ & Female $(n=94)$ & & & \\
\hline Overall liking ${ }^{1}$ & $4.77(1.51)^{b}$ & $3.86(1.76)^{\mathrm{a}}$ & $5.30(1.31)^{b}$ & 22.083 & $<0.001$ \\
\hline Overall appearance $^{1}$ & $3.96(1.59)^{\mathrm{a}, \mathrm{b}}$ & $3.59(1.61)^{\mathrm{a}}$ & $4.58(1.39)^{b}$ & 11.060 & $<0.001$ \\
\hline Texture $^{1}$ & $5.00(1.50)$ & $4.72(1.50)$ & $5.15(1.35)$ & 2.239 & 0.109 \\
\hline Color $^{1}$ & $3.73(1.64)^{\mathrm{a}}$ & $3.55(1.73)^{\mathrm{a}}$ & $4.54(1.75)^{b}$ & 8.532 & $<0.001$ \\
\hline Taste $^{1}$ & $4.35(1.72)^{\mathrm{a}}$ & $3.81(1.77)^{\mathrm{a}}$ & $5.26(1.40)^{b}$ & 20.931 & $<0.001$ \\
\hline Mouth feel $^{1}$ & $5.08(1.50)$ & $4.50(1.65)$ & $5.08(1.42)$ & 4.009 & 0.019 \\
\hline Amount of fruit flavor ${ }^{2}$ & $4.42(1.27)$ & $4.2(1.24)$ & $4.08(0.79)$ & 1.129 & 0.325 \\
\hline Amount of sweetness $^{3}$ & $4.04(0.66)$ & $3.99(1.30)$ & $4.03(0.86)$ & 0.043 & 0.958 \\
\hline
\end{tabular}

${ }^{1}$ Scale: 1 = Dislike very much 2 = Dislike moderately $3=$ Dislike slightly 4 = Neither like not dislike $5=$ Like slightly $6=$ Like moderately $7=$ Like very much; ${ }^{2}$ Scale: 1 = Much too little 2 = Moderately too little $3=$ Slightly too little $4=$ Just about right $5=$ Slightly too much $6=$ Moderately too much $7=$ Much too much; ${ }^{3}$ Scale: $1=$ Much too tart $2=$ Moderately tart $3=$ Slightly tart $4=$ Just about right $5=$ Slightly too sweet $6=$ Moderately too sweet $7=$ Much too sweet; ${ }^{\mathrm{ab}}$ Different letters represent statistical differences between the groups.

Table 3. Attribute comparison of mixed berry-flavored applesauce (by group).

\begin{tabular}{|c|c|c|c|c|c|}
\hline \multirow{2}{*}{ Attributes } & \multicolumn{2}{|c|}{ Adults } & \multirow{2}{*}{ Kids $(n=106)$} & \multirow{2}{*}{$F$ value } & \multirow{2}{*}{$p$ value } \\
\hline & Male $(n=26)$ & Female $(\mathrm{n}=94)$ & & & \\
\hline Overall liking $^{1}$ & $5.12(1.84)^{b}$ & $4.31(1.70)^{\mathrm{a}}$ & $5.72(1.53)^{b}$ & 18.437 & $<0.001$ \\
\hline Overall appearance ${ }^{1}$ & $4.81(1.86)^{\mathrm{ab}}$ & $4.22(1.60)^{\mathrm{a}}$ & $5.42(1.29)^{\mathrm{b}}$ & 16.034 & $<0.001$ \\
\hline Texture $^{1}$ & $5.31(1.76)^{b}$ & $4.60(1.45)^{\mathrm{a}}$ & $5.43(1.30)^{b}$ & 9.077 & $<0.001$ \\
\hline Color $^{1}$ & $4.85(1.89)^{\mathrm{a}}$ & $4.09(1.75)^{\mathrm{a}}$ & $5.70(1.45)^{b}$ & 24.327 & $<0.001$ \\
\hline Taste $^{1}$ & $5.35(1.52)^{b}$ & $4.39(1.71)^{\mathrm{a}}$ & $5.76(1.40)^{b}$ & 19.733 & $<0.001$ \\
\hline Mouth feel ${ }^{1}$ & $5.46(1.56)^{b}$ & $4.71(1.53)^{\mathrm{a}}$ & $5.44(1.27)^{b}$ & 7.370 & 0.001 \\
\hline Amount of fruit flavor ${ }^{2}$ & $4.31(1.05)$ & $4.36(1.06)$ & $4.08(0.80)$ & 2.414 & 0.092 \\
\hline Amount of sweetness ${ }^{3}$ & $3.92(0.94)$ & 4.13 (1.29) & $3.99(0.67)$ & 0.667 & 0.514 \\
\hline
\end{tabular}

${ }^{1}$ Scale: 1 = Dislike very much 2 = Dislike moderately 3 = Dislike slightly 4 = Neither like not dislike $5=$ Like slightly $6=$ Like moderately $7=$ Like very much ${ }^{2}$ Scale: 1 = Much too little $2=$ Moderately too little $3=$ Slightly too little $4=$ Just about right $5=$ Slightly too much $6=$ Moderately too much $7=$ Much too much; ${ }^{3}$ Scale: 1 = Much too tart 2 = Moderately tart $3=$ Slightly tart $4=$ Just about right $5=$ Slightly too sweet $6=$ Moderately too sweet 7 = Much too sweet; ${ }^{\mathrm{ab}}$ Different letters represent statistical differences between the groups.

Table 4. Attribute comparison of strawberry-flavored applesauce (by group).

\begin{tabular}{|c|c|c|c|c|c|}
\hline \multirow{2}{*}{ Attributes } & \multicolumn{2}{|c|}{ Adults } & \multirow{2}{*}{ Kids $(n=106)$} & \multirow{2}{*}{$F$ value } & \multirow{2}{*}{$p$ value } \\
\hline & Male $(\mathrm{n}=26)$ & Female $(n=94)$ & & & \\
\hline Overall liking ${ }^{1}$ & $4.73(1.78)$ & $4.44(1.73)$ & $4.08(1.95)$ & 1.753 & 0.176 \\
\hline Overall appearance $^{1}$ & $5.35(1.33)$ & $5.10(1.31)$ & $4.97(1.67)$ & 0.688 & 0.504 \\
\hline Appearance of texture ${ }^{1}$ & $5.35(1.38)$ & $5.15(1.29)$ & $5.08(1.36)$ & 0.438 & 0.646 \\
\hline Appearance of color ${ }^{1}$ & $5.31(1.41)$ & $5.01(1.51)$ & $4.86(1.89)$ & 0.778 & 0.460 \\
\hline Taste of apple sauce ${ }^{1}$ & $4.81(1.74)$ & $4.59(1.80)$ & $4.10(1.93)$ & 2.436 & 0.090 \\
\hline Mouth feel of apple sauce ${ }^{1}$ & $5.27(1.46)$ & $4.93(1.62)$ & $4.76(1.71)$ & 1.021 & 0.362 \\
\hline Amount of fruit flavor ${ }^{2}$ & $3.88(1.03)$ & $4.18(0.97)$ & $3.80(1.21)$ & 3.070 & 0.048 \\
\hline Amount of sweetness $^{3}$ & $3.81(0.57)$ & $3.95(1.06)$ & $3.77(1.16)$ & 0.681 & 0.507 \\
\hline
\end{tabular}

${ }^{1}$ Scale: 1 = Dislike very much 2 = Dislike moderately $3=$ Dislike slightly 4 = Neither like not dislike $5=$ Like slightly $6=$ Like moderately $7=$ Like very much; ${ }^{2}$ Scale: 1 = Much too little 2 = Moderately too little 3 = Slightly too little 4 = Just about right 5 = Slightly too much $6=$ Moderately too much 7 = Much too much;

${ }^{3}$ Scale: 1 = Much too tart 2 = Moderately tart 3 = Slightly tart $4=$ Just about right $5=$ Slightly too sweet $6=$ Moderately too sweet $7=$ Much too sweet. 
Table 5. Comparison of attributes within groups (by flavor).

\begin{tabular}{|c|c|c|c|c|c|c|c|c|c|c|c|c|}
\hline \multirow{3}{*}{ Attributes } & \multicolumn{8}{|c|}{ Adults } & \multirow{2}{*}{\multicolumn{4}{|c|}{ Kids }} \\
\hline & \multicolumn{4}{|c|}{ Male } & \multicolumn{4}{|c|}{ Female } & & & & \\
\hline & Tropical & $\begin{array}{l}\text { Mixed } \\
\text { berry }\end{array}$ & Strawberry & $\begin{array}{c}F \\
\text { value }\end{array}$ & Tropical & $\begin{array}{l}\text { Mixed } \\
\text { berry }\end{array}$ & Strawberry & $\begin{array}{c}F \\
\text { value }\end{array}$ & Tropical & $\begin{array}{l}\text { Mixed } \\
\text { berry }\end{array}$ & Strawberry & $\begin{array}{c}F \\
\text { value }\end{array}$ \\
\hline Liking $^{1}$ & $\begin{array}{c}4.77 \\
(1.51)\end{array}$ & $\begin{array}{c}5.12 \\
(1.84)\end{array}$ & $\begin{array}{c}4.73 \\
(1.78)\end{array}$ & 0.397 & $\begin{array}{c}3.86 \\
(1.76)\end{array}$ & $\begin{array}{c}4.31 \\
(1.70)\end{array}$ & $\begin{array}{c}4.44 \\
(1.73)\end{array}$ & 2.860 & $\begin{array}{c}5.3 \\
(1.31)^{\mathrm{a}}\end{array}$ & $\begin{array}{c}5.72 \\
(1.53)^{\mathrm{a}}\end{array}$ & $\begin{array}{c}4.08 \\
(1.95)^{\mathrm{b}}\end{array}$ & $29.475^{* * *}$ \\
\hline Appearance $^{1}$ & $\begin{array}{c}3.96 \\
(1.59)^{\mathrm{a}}\end{array}$ & $\begin{array}{c}4.81 \\
(1.86)^{\mathrm{ab}}\end{array}$ & $\begin{array}{c}5.35 \\
(1.33)^{\mathrm{b}}\end{array}$ & $4.925^{*}$ & $\begin{array}{c}3.59 \\
(1.61)^{\mathrm{a}}\end{array}$ & $\begin{array}{c}4.22 \\
(1.60)^{\mathrm{b}}\end{array}$ & $\begin{array}{c}5.10 \\
(1.31)^{\mathrm{c}}\end{array}$ & $23.592^{* * *}$ & $\begin{array}{c}4.58 \\
(1.39)^{\mathrm{a}}\end{array}$ & $\begin{array}{c}5.42 \\
(1.29)^{\mathrm{b}}\end{array}$ & $\begin{array}{c}4.97 \\
(1.67)^{\mathrm{ab}}\end{array}$ & $8.768^{* * *}$ \\
\hline Texture $^{1}$ & $\begin{array}{c}5.00 \\
(1.50)\end{array}$ & $\begin{array}{c}5.31 \\
(1.76)\end{array}$ & $\begin{array}{c}5.35 \\
(1.38)\end{array}$ & 0.387 & $\begin{array}{c}4.72 \\
(1.50)^{\mathrm{ab}}\end{array}$ & $\begin{array}{c}4.60 \\
(1.45)^{\mathrm{a}}\end{array}$ & $\begin{array}{c}5.15 \\
(1.29)^{\mathrm{b}}\end{array}$ & $3.948^{*}$ & $\begin{array}{c}5.15 \\
(1.35)\end{array}$ & $\begin{array}{c}5.43 \\
(1.30)\end{array}$ & $\begin{array}{c}5.08 \\
(1.36)\end{array}$ & 2.118 \\
\hline Color $^{1}$ & $\begin{array}{c}3.73 \\
(1.64)^{\mathrm{a}}\end{array}$ & $\begin{array}{c}4.85 \\
(1.89)^{\mathrm{b}}\end{array}$ & $\begin{array}{c}5.31 \\
(1.41)^{\mathrm{b}}\end{array}$ & $6.221^{* *}$ & $\begin{array}{c}3.55 \\
(1.73)^{\mathrm{a}}\end{array}$ & $\begin{array}{c}4.09 \\
(1.75)^{\mathrm{a}}\end{array}$ & $\begin{array}{c}5.01 \\
(1.51)^{\mathrm{b}}\end{array}$ & $18.442^{* * *}$ & $\begin{array}{c}4.54 \\
(1.75)^{\mathrm{a}}\end{array}$ & $\begin{array}{c}5.70 \\
(1.45)^{\mathrm{b}}\end{array}$ & $\begin{array}{c}4.86 \\
(1.89)^{\mathrm{a}}\end{array}$ & $13.066^{* * *}$ \\
\hline Taste $^{1}$ & $\begin{array}{c}4.35 \\
(1.72)\end{array}$ & $\begin{array}{c}5.35 \\
(1.52)\end{array}$ & $\begin{array}{c}4.81 \\
(1.74)\end{array}$ & 2.351 & $\begin{array}{c}3.81 \\
(1.77)^{\mathrm{a}}\end{array}$ & $\begin{array}{c}4.39 \\
(1.71)^{\mathrm{ab}}\end{array}$ & $\begin{array}{c}4.59 \\
(1.80)^{\mathrm{b}}\end{array}$ & $4.970^{* *}$ & $\begin{array}{c}5.26 \\
(1.40)^{\mathrm{a}}\end{array}$ & $\begin{array}{c}5.76 \\
(1.40)^{\mathrm{a}}\end{array}$ & $\begin{array}{c}4.10 \\
(1.93)^{\mathrm{b}}\end{array}$ & $30.224^{* * *}$ \\
\hline Mouth feel ${ }^{1}$ & $\begin{array}{c}5.08 \\
(1.50)\end{array}$ & $\begin{array}{c}5.46 \\
(1.56)\end{array}$ & $\begin{array}{c}5.27 \\
(1.46)\end{array}$ & 0.426 & $\begin{array}{c}4.50 \\
(1.65)\end{array}$ & $\begin{array}{c}4.71 \\
(1.53)\end{array}$ & $\begin{array}{c}4.93 \\
(1.62)\end{array}$ & 1.660 & $\begin{array}{c}5.08 \\
(1.42)^{\mathrm{ab}}\end{array}$ & $\begin{array}{c}5.44 \\
(1.27)^{\mathrm{a}}\end{array}$ & $\begin{array}{c}4.76 \\
(1.71)^{\mathrm{b}}\end{array}$ & $5.608^{* *}$ \\
\hline Fruit flavor $^{2}$ & $\begin{array}{c}4.42 \\
(1.27)\end{array}$ & $\begin{array}{c}4.31 \\
(1.05)\end{array}$ & $\begin{array}{c}3.88 \\
(1.03)\end{array}$ & 1.658 & $\begin{array}{c}4.20 \\
(1.24)\end{array}$ & $\begin{array}{c}4.36 \\
(1.06)\end{array}$ & $\begin{array}{c}4.18 \\
(0.97)\end{array}$ & 0.765 & $\begin{array}{c}4.08 \\
(0.79)\end{array}$ & $\begin{array}{c}4.08 \\
(0.80)\end{array}$ & $\begin{array}{c}3.80 \\
(1.21)\end{array}$ & 3.012 \\
\hline Sweetness ${ }^{3}$ & $\begin{array}{c}4.04 \\
(0.66)\end{array}$ & $\begin{array}{c}3.92 \\
(0.94)\end{array}$ & $\begin{array}{c}3.81 \\
(0.57)\end{array}$ & 0.636 & $\begin{array}{c}3.99 \\
(1.30)\end{array}$ & $\begin{array}{c}4.13 \\
(1.29)\end{array}$ & $\begin{array}{c}3.95 \\
(1.06)\end{array}$ & 0.563 & $\begin{array}{c}4.03 \\
(0.86)\end{array}$ & $\begin{array}{c}3.99 \\
(0.67)\end{array}$ & $\begin{array}{c}3.77 \\
(1.16)\end{array}$ & 2.386 \\
\hline
\end{tabular}

${ }^{1}$ Scale: 1 = Dislike very much 2 = Dislike moderately $3=$ Dislike slightly $4=$ Neither like not dislike $5=$ Like slightly $6=$ Like moderately $7=$ Like very much; ${ }^{2}$ Scale: 1 = Much too little 2 = Moderately too little $3=$ Slightly too little $4=$ Just about right $5=$ Slightly too much $6=$ Moderately too much $7=$ Much too much; ${ }^{3}$ Scale: 1 = Much too tart $2=$ Moderately tart $3=$ Slightly tart $4=$ Just about right $5=$ Slightly too sweet $6=$ Moderately too sweet $7=$ Much too sweet;

${ }^{\mathrm{ab}}$ Different letters represent statistical differences between the samples; ${ }^{*} p<0.05 ;{ }^{* *} p<0.01 ;{ }^{* * * *} p<0.001$.

expectancy of Americans during the $20^{\text {th }}$ century increased by almost 30 years, with much of that increase attributed to the significant decrease in infant and childhood mortality rates since 1900 . Unfortunately, that trend could be reversed during the $21^{\text {st }}$ century if society cannot tackle the primary health issue currently facing American children-the childhood obesity epidemic [21].

Encouraging individuals to make healthier food choices can be made easier by developing and testing newer, healthier foods that are accessible, affordable and satisfying to the consumer. This study-which evaluated the sensory characteristics of, and consumer preference for, tropical-, mixed berry- and strawberry-flavored vegetable-infused applesauces_-showed that developing such products is reasonable.

This study expects a potential contribution of vegetable-infused applesauce in terms of increasing children's vegetable consumption as a stealth health food. "Stealth health" foods do not solve the problem of educating children about the value of eating vegetables to their long-term health. However, as a strategy to use in concert with nutrition education, formulations of popular foods with more healthful ingredients will immediately provide a benefit of increasing consumption of valuable nutrients. Although children know that fruit and vegetables are healthful foods, thus, having such foods is desirable, their preference are not congruent with their perceptions mainly due to taste $[1,22]$. Leading children to get familiar with vegetable taste unconsciously from vegetableinfused fruit-flavor applesauce will make children be less resistant to typical bitter taste of vegetables, which can be considered a long-term indirect effect of a vegetableinfused applesauce.

Interestingly, kids showed significantly higher preference for mixed berry- and tropical-flavored applesauce and relatively lower preference for strawberry-flavored applesauce than adults did. It would be because children are already familiar with berry flavors in other food products (i.e., berry-flavored cereals and jams). Marketers or foodservice managers need to consider serving different types of vegetable-infused fruit-flavored applesauce according to different age group.

Future studies may consider applying such products in real fields and investigate consumer's actual buying behavior. As a longitudinal study, it would be interesting to see any change of children's vegetable consumption pattern for several years in order to investigate if vegetableinfused applesauce plays a role of stealth foods by exposing children to vegetable taste. This study was conducted with only middle school students. Future studies need to investigate younger children's perceptions as 
well.

\section{Acknowledgements}

This study was funded by Knouse Foods; however, analysis and final results of this study were in no way influenced by representatives of the company. Data collection and analysis software were provided by Compusense (Guelph, Ontario, Canada). We thank Samantha A. Ream and Kimberly C. Snyder for their assistance in collecting data.

\section{REFERENCES}

[1] C. Noble, M. Corney, A. Eves, M. Kipps and M. Lumbers, "Food Choice and School Meals: Primary School Children's Perceptions of the Healthiness of Foods and the Nutritional Implications of Food Choices,” International Journal of Hospitality Management, Vol. 19, No. 4, 2000, pp. 413-432. doi:10.1016/S0278-4319(00)00038-4

[2] C. Ogden and M. Carroll, "Prevalence of Obesity among Children and Adolescents: United State, Trends 19631965 through 2007-2008,” 2010.

http://www.nccpeds.com/ContinuityModules-Fall/Obesit y\%20Trends.pdf

[3] D. S. Freedman, W. H. Dietz, S. R. Srinivasan and G. S. Berenson, "The Relation of Overweight to Cardiovascular Risk Factors among Children and Adolescents: The Bogalusa Heart Study,” Pediatrics, Vol. 103, No. 6, 1999, pp. 1175-1182. doi:10.1542/peds.103.6.1175

[4] US Surgeon General, "Overweight and Obesity: Health Consequences,” 2006.

http://www.surgeongeneral.gov/topics/obesity/calltoactio n/fact_consequences.html

[5] J. J. Reilly and J. Kelly, "Long-Term Impact of Overweight and Obesity in Childhood and Adolescence on Morbidity Premature Mortality in Adulthood: Systematic Review,” International Journal of Obesity, Vol. 35, No. 7, 2011, pp. 891-898. doi:10.1038/ijo.2010.222

[6] S. D. Stovitz, P. J. Hannan, L. A. Lytle, E. W. Demerath, M. A. Pereira, et al., "Child Height and the Risk of YoungAdult Obesity," American Journal of Preventive Medicine, Vol. 38, No. 1, 2010, pp. 74-77. doi:10.1016/j.amepre.2009.09.033

[7] United States Department of Agriculture, “Choose a Food Group,” 2011a. http://www.choosemyplate.gov/food-groups/

[8] P. M. Guenther, K. W. Dodd, J. Reedy and S. M. KrebsSmith, "Most Americans Eat Much Less than Recommended Amounts of Fruits and Vegetables," Journal of the American Dietetic Association, Vol. 106, No. 9, 2006, pp. 1371-1379. doi:10.1016/j.jada.2006.06.002
[9] Centers for Disease Control and Preventions, "State Indicator Report on Fruits and Vegetables,” 2009. http://www.fruitsandveggiesmatter.gov/health_profession als/statereport.html

[10] B. A. Lorson, H. R. Melgar-Quinonez and C. A. Taylor, "Correlates of Fruit and Vegetable Intakes in US Children," Journal of American Dietetic Association, Vol. 109, No. 3, 2009, pp. 474-478. doi:10.1016/j.jada.2008.11.022

[11] B. A. Dennison, H. L. Rockwell and S. L. Baker, "Fruit and Vegetable Intake in Young Children," Journal of the American College of Nutrition, Vol. 17, No. 4, 1998, pp. 371-378.

[12] K. A. Munoz, S. M. Krebs-Smith, R. Ballard-Barbash and L. E. Cleveland, "Food Intakes of US Children and Adolescents Compared with Recommendations," Pediatrics, Vol. 100, No. 3, 1997, pp. 323-329. doi:10.1542/peds.100.3.323

[13] R. D. Srinath and M. B. Katan, "Diet, Nutrition and the Prevention of Hypertension and Cardiovascular Diseases,” Public Health Nutrition, Vol. 7, No. 1A, 2004, pp. 167-186.

[14] K. A. Steinmetz and J. D. Potter, "Vegetables, Fruit, and Cancer Prevention: a Review,” Journal of American Dietetic Association, Vol. 96, No. 10, pp. 1027-1039. doi:10.1016/S0002-8223(96)00273-8

[15] Let's Move Salad Bars to Schools, "Salad Bars to Schools,” 2011. http://saladbars2schools.org/

[16] J. Boyer and R. H. Liu, "Apple Phytochemicals and Their Health Benefits,” Nutrition Journal, Vol. 3, No. 5, 2004, pp. 1-15.

[17] S. Busch, “Nutrition Facts in Applesauce,” 2011. http://www.livestrong.com/article/530239-nutrition-factsin-applesauce/

[18] United States Department of Agriculture, “Applesauce Nutrition List,” 2011b. http://ndb.nal.usda.gov/ndb/foods/list

[19] C. Kylstra, “6 Stealth Health Foods,” 2009. www.MensHealth.com

[20] A. M. Miller, "Stealth Health: How the Culinary Institute of America is Brining Flavor to Healthy Meals for Kids," Childhood Obesity, 2011, Vol. 7, No. 3, pp. 165-168.

[21] S. J. Olshansky, D. J. Passaro, R. C. Hershow, J. Layden, B. A. Carnes, et al., "A Potential Decline in Life Expectancy in the United States in the $21^{\text {st }}$ Century," New England Journal of Medicine, Vol. 352, No. 11, 2005, pp. 1138-1145. doi:10.1056/NEJMsr043743

[22] J. L. P. Protudjer, G. Marchessault, A. L. Kozyrskyj and A. B. Becker, "Children's Perceptions of Healthful Eating and Physical Activity," Canadian Journal of Dietetic Practice and Research, Vol. 71, No. 1, 2010, pp. 19-23. doi:10.3148/71.1.2010.19 